\title{
ON THE BIAS OF TERMINAL BASED GAIN AND OFFSET ESTIMATION USING THE ADC HISTOGRAM TEST METHOD
}

\author{
F.Corrêa Alegria ${ }^{1)}$, Nestor Michael C. Tiglao ${ }^{2)}$ \\ 1) Instituto Superior Técnico and Instituto de Telecomunicações, Av. Rovisco Pais 1, 1049-001 Lisbon, Portugal \\ (\falegria@lx.it.pt, +351218418376) \\ 2) Instituto Superior Técnico, Lisbon, Portugal and EEEI, University of the Philippines (nestor.tiglao@inesc-id.pt)
}

\begin{abstract}
The Histogram Test method is a popular technique in analog-to-digital converter (ADC) testing. The presence of additive noise in the test setup or in the ADC itself can potentially affect the accuracy of the test results. In this study, we demonstrate that additive noise causes a bias in the terminal based estimation of the gain but not in the estimation of the offset. The estimation error is determined analytically as a function of the sinusoidal stimulus signal amplitude and the noise standard deviation. We derive an exact but computationally difficult expression as well as a simpler closed form approximation that provides an upper bound of the bias of the terminal based gain. The estimators are validated numerically using a Monte Carlo procedure with simulated and experimental data.
\end{abstract}

Keywords: analog to digital converter, histogram test method, estimator bias, terminal based, gain, offset.

(c) 2011 Polish Academy of Sciences. All rights reserved

\section{Introduction}

The Histogram Test (or Code Density Test) is widely used in analog-to-digital converter (ADC) testing [1]-[3]. This method allows the estimation of different ADC parameters, namely the transition voltages, code bin widths integral non-linearity, differential nonlinearity, gain, and offset error. The estimators for these parameters are affected by the nonideal effects of the test setup or in the ADC itself, among which are additive noise [3]-[5], phase noise and jitter [6]-[8], stimulus signal distortion [3], and frequency error [9], [10].

In this paper, we study the influence of additive noise on the estimation error of the ADC gain and offset error through numerical simulations as well as experimental measurements. This is useful not only to statistically characterize the results obtained when testing an ADC but also to be able to correct the estimates made. The latter is possible when the amount of additive noise present is known or can be determined. This source of uncertainty needs to be taken into account when computing the measurement uncertainty of ADC-based instruments [11], [12].

This paper is an update of [13] where we have added experimental results that validate the analytical expression presented.

In section 2, we introduce the terminal based definition of gain and offset error. In section 3 , we derive the errors of the estimators. In section 4, we validate the numerical results from Monte Carlo simulation and in section 5 we validate the estimators through experimental measurements. Finally, we draw some conclusions in section 6 . 


\section{Terminal Based Gain and Offset Error}

The purpose of an ADC is to convert the values of a current or voltage present at the input, which is a continuous variable, into a digital word that should represent that input. That relationship, between the input variable and output digital words (or codes) is known as the $\mathrm{ADC}$ transfer function and is determined by the ADC manufacturer [14]. In the rest of the text we will consider that the input variable is a voltage. There are different types of transfer functions. One of them, used with bipolar ADCs, is the mid-riser (also known as "with no true zero") which is represented in Fig. 1. Variable $n_{b}$ represents the ADC number of bits and FS the full scale voltage.

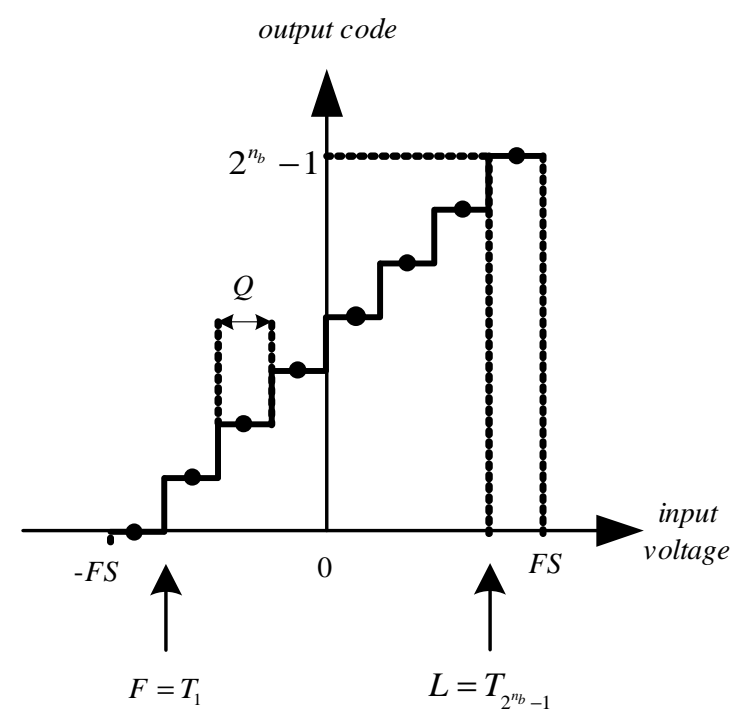

Fig. 1. Illustration of the transfer function of a bipolar ADC. This type of transfer function is known as mid-riser or "with no true zero".

Each output code corresponds to a range of input voltage values (horizontal lines). Given an output code one cannot determine exactly which was the input voltage at the time of the sampling and posterior analog-to-digital conversion. It is conventional to adopt the middle point of the ranges mentioned as the value of the input voltage for a given output code (black circles).

The transition voltages, $T_{k}$, define the ADC transfer function, that is, the relation between input voltage and output code, $k$. For an ideal ADC, the transition voltages of the transfer function, defined as in Fig. 1, are

$$
T_{k}^{i d e a l}=-F S+k \cdot Q
$$
by

They are equally spaced by an amount $Q$ given, from the definition of the transfer function,

$$
Q=\frac{2 \cdot F S}{2^{n} b}
$$

In an actual $\mathrm{ADC}$, the real transition voltages will be different from the ideal ones. To express those differences several parameters are used. Two of those are the ADC gain and offset error. They can be defined in different ways. Two of the most used are the Terminal Based Definition and the Independently Based Definition [1]. In this paper we will focus our 
attention on the first one. According to the Terminal Based Definition, the offset error plus the product of the gain by the first and last real transition voltages, results in the first and last ideal transition voltages respectively. Hence the designation "Terminal Based" refers to the fact that the definition is based on the extremes of the transfer function, that is, on the value of the first (lowest) and last (highest) transition voltages. So that the gain $(G)$ and offset error $(O)$ satisfy the definition if they are computed with the following expressions [15]:

$$
G=\frac{L_{\text {ideal }}-F_{i d e a l}}{L-F} \text { and } O=F_{i d e a l}-G \cdot F .
$$

To simplify the notation, we introduced the variables $F=T_{1}$ and $L=T_{2^{2_{b}}-1}$.

When testing an ADC with the Standard Histogram Test we obtain an estimate of the transition voltages (not the real transition voltages). From those estimates we can compute the estimated ADC gain and offset error using

$$
\widehat{G}=\frac{L_{\text {ideal }}-F_{i d e a l}}{\widehat{L}-\widehat{F}} \text { and } \widehat{O}=F_{i d e a l}-\widehat{G} \cdot \widehat{F} \text {. }
$$

The "hat" symbol over the variables means that they are an estimate and not the actual values for the ADC under test.

\section{Derivation Of The Estimation Bias}

The presence of additive noise, which is a stochastic perturbation of the voltage sampled by the ADC, causes all the quantities estimated with the Histogram Method to be also stochastic variables. This is so for the estimated ADC gain and offset error given by (4) since they are determined from the estimated values of the first $(F)$ and last $(L)$ transition voltages.

The expected value of the estimates given by (4) can be computed from the statistical properties of $\widehat{F}$ and $\hat{L}$. This can be done using

$$
\mathrm{E}\{g\} \simeq g+\frac{1}{2}\left(\frac{\partial^{2} g}{\partial x^{2}} \sigma_{x}^{2}+\frac{\partial^{2} g}{\partial x \partial y} r \sigma_{x} \sigma_{y}+\frac{\partial^{2} g}{\partial y^{2}} \sigma_{y}^{2}\right),
$$

where $g$ is a function of two random variables $x$ and $y$ with correlation coefficient $r$ [16], p. 156. The function and its derivatives are evaluated at $x=\mathrm{E}\{x\}$ and $y=\mathrm{E}\{y\}$. In this paper, however only the first term in (5) is going to be used. This leads to a poorer approximation, however, since the analytical expressions for expected values of $x$ and $y$ ( $\hat{F}$ and $\hat{L}$ in the context of this paper) that are going to be used are themselves coarse approximation for their exact value, it would be unnecessary to consider the second term in (5). This choice will be numerically validated later using Monte Carlo simulations.

The expected value of the function will thus be considered equal to the function of the expected value. Using (4) leads to

$$
\mathrm{E}\{\widehat{G}\}=\frac{L_{\text {ideal }}-F_{\text {ideal }}}{\mathrm{E}\{\widehat{L}\}-\mathrm{E}\{\widehat{F}\}}, \mathrm{E}\{\widehat{O}\}=F_{\text {ideal }}-\mathrm{E}\{\widehat{G}\} \cdot \mathrm{E}\{\widehat{F}\} .
$$

In [17], the amount of overdrive to use in order to minimize the error in the estimation of the transition voltages due to additive noise was studied. There, an expression for the computation of the expected value of the transition voltages estimated with the Histogram Method has been derived, 


$$
\mathrm{E}\left\{\widehat{U}_{k}\right\} \approx-\cos \left\{\int_{0}^{\pi}\left[\frac{1}{2}+\frac{1}{2} \operatorname{erf}\left(\frac{U_{k}+\cos (\varphi)}{\sqrt{2} \sigma_{n}}\right)\right] \mathrm{d} \varphi\right\},
$$

where $U_{k}$ and $\sigma_{n}$ are the normalized values of transition voltage and additive noise standard deviation. They are obtained from the real values (voltages) by subtracting the stimulus signal offset $C$ (just in the case of the transition voltages) and dividing by its amplitude, $A$. These normalized values make the derivations clearer and can easily be converted back to voltages.

Note that the expected value of the estimated transition voltage, $\widehat{U_{k}}$, given by (7) depends on the actual transition voltage $U_{k}$. It is, however, safe to use the ideal value of the transition voltage in place of the actual value which is unknown, since typically they are similar.

In order to simplify the presentation, it is assumed we are dealing with an ADC that has a mid-riser transfer function like the one depicted in Fig. 1. In this type of transfer function the transition voltages are symmetric which eases the analytical derivations. One has, thus,

$$
L_{i d e a l}=-F_{i d e a l}=F S-Q \text {, }
$$

where (1) and (2) were used. Using this it is possible to write (6) as

$$
\begin{aligned}
& \mathrm{E}\{\widehat{G}\}=\frac{2(F S-Q)}{\mathrm{E}\{\widehat{L}\}-\mathrm{E}\{\widehat{F}\}} \\
& \mathrm{E}\{\widehat{O}\}=-F S+Q-\mathrm{E}\{\widehat{G}\} \cdot \mathrm{E}\{\widehat{F}\} .
\end{aligned}
$$

Also, expression (7) is odd in relation to $U_{k}$. Leading to

$$
\mathrm{E}\{\widehat{L}\}=-\mathrm{E}\{\widehat{F}\} \text {. }
$$

Using (10) it is possible to write (9) as,

$$
\mathrm{E}\{\widehat{G}\}=\frac{F S-Q}{\mathrm{E}\{\widehat{L}\}} \text { and } \mathrm{E}\{\widehat{O}\}=0 .
$$

This shows immediately that the offset error estimator is unbiased since its expected value is equal to its ideal value which is zero.

Inserting (7) into (11) and using the ideal value for the last transition voltage in place of the actual value in (7), leads to

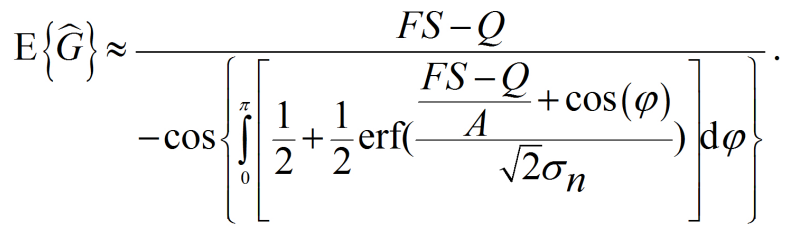

In [17] a careful analysis of the dependence of (7) on the values of the transition voltage and the additive noise standard deviation was carried out. It was possible to determine the maximum value of the estimation error of the transition voltages for a given additive noise standard deviation. The expression obtained was

where

$$
\begin{gathered}
e_{\widehat{U}} \max \\
\approx \frac{\sigma_{n}}{5} \quad \text { for } \quad \sigma_{n}<0.1 \\
e_{\widehat{U}} \triangleq \mu_{\overparen{U}}-U
\end{gathered}
$$


This is valid for values of normalized noise standard deviation lower than 0.1 which is equivalent to having a noise standard deviation lower than $10 \%$ of the stimulus signal amplitude, a situation generally encountered in practice. Using this, an approximate expression for the upper bound of the expected value of the normalized transition voltage is

$$
\mathrm{E}\{\widehat{U}\}<U+\frac{\sigma_{n}}{5} \quad \text { for } \quad \sigma_{n}<0.1
$$

Using (15) in (11) leads to

$$
\mathrm{E}\{\widehat{G}\}<\frac{F S-Q}{F S-Q+\frac{\sigma_{n v}}{5}} .
$$

Considering that the ideal code bin width $(Q)$ is much smaller than the ADC full scale $(F S)$, it is possible to write (16) as

$$
\mathrm{E}\{\widehat{G}\}<\frac{5}{5+\frac{\sigma_{n v}}{F S}} .
$$

The relative error of the estimator, defined by

$$
\varepsilon_{\widehat{G}} \triangleq\left|\frac{\mathrm{E}\{\widehat{G}\}-G_{\text {ideal }}}{G_{\text {ideal }}}\right|=|\mathrm{E}\{\widehat{G}\}-1|,
$$

is thus

$$
\varepsilon_{\widehat{G}}<\frac{1}{5} \frac{\sigma_{n v}}{F S}
$$

This expression allows the determination of an upper bound on the relative error in the estimation of the ADC gain using the Histogram Method. It can be seen that this upper bound is proportional to the standard deviation of the additive noise $\left(\sigma_{n v}\right)$ relative to the ADC full scale $(F S)$.

\section{Numerical Validation}

In order to validate the derivations presented here and the approximations made, namely the substitution of the expected value of a function by the function of the expected value of its argument in (6), the use of expression (7), derived in [17] and which is in itself an approximate expression and the upper bound on the estimation error of the transition voltages made in (13), a Monte Carlo procedure was used. It consists in repeatedly simulating a sinusoidal stimulus signal corrupted by additive noise and using the Histogram Test to estimate the terminal based gain and offset of a simulated ADC.

In Fig. 2 the expected value of the estimated ADC gain is depicted as a function of the normalized additive noise standard deviation (black circles). The vertical bars represent the confidence interval for a $99.9 \%$ confidence level obtained with 1000 repetitions of the Histogram Test. It can be seen that the results conform to expression (12) which was derived here and which can be used to analytically determine the estimation error. The dashed line is the representation of expression (15) which gives a bound for the estimation error and which is much easier to use than (12). 


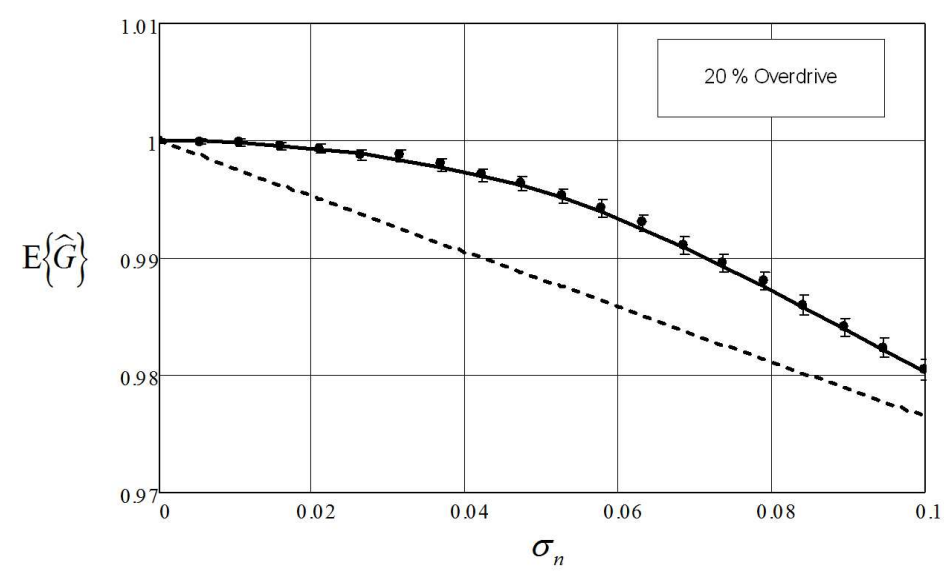

Fig. 2. Expected value of the estimated ADC gain as a function of the normalized additive noise standard deviation for a stimulus signal amplitude of $1.2 \mathrm{~V}$. The circles represent the values obtained numerically. The vertical bars represent the confidence intervals for a $99.9 \%$ confidence level. The solid line is the representation of expression (12) and the dashed line is the representation of (17).

In Fig. 3 the same information is depicted but now using 5\% overdrive. Again the numerical results obtained are in conformance with the analytical expressions presented here.

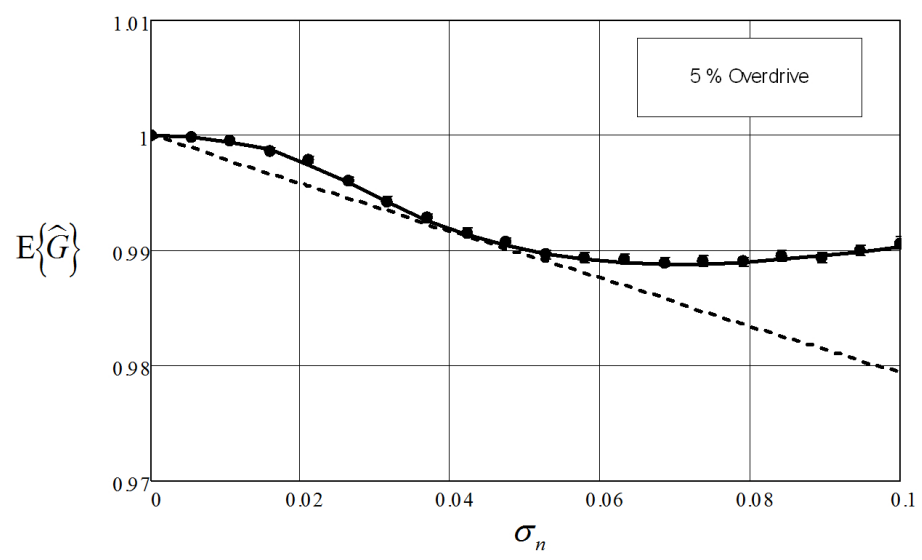

Fig. 3. Expected value of the estimated ADC gain as a function of the normalized additive noise standard deviation for a stimulus signal amplitude of $1.05 \mathrm{~V}$. The circles represent the values obtained numerically. The vertical bars represent the confidence intervals for a $99.9 \%$ confidence level. The solid line is the representation of expression (12) and the dashed line is the representation of (17).

Table 1 lists the values of the parameters of the test setup used in the numerical simulation.

Table 1. List of test setup parameters used in the numerical validation.

\begin{tabular}{cc}
\hline Test Parameter & Value \\
\hline Number of Bits of the ADC $\left(n_{b}\right)$ & 8 \\
\hline ADC Full Scale $(F S)$ & $1 \mathrm{~V}$ \\
\hline Sinusoidal Stimulus Amplitude $(A)$ & $1.05 \mathrm{~V}$ and $1.2 \mathrm{~V}$ \\
\hline Sinusoidal Stimulus Offset $(C)$ & 0 \\
\hline Number of Samples $(M)$ & 1000 \\
\hline Additive Noise Standard Deviation $\left(\sigma_{n v}\right)$ & 0 to $0.1 \times \mathrm{A}$ \\
\hline Number of Repetitions & 1000 \\
\hline Confidence Level & $99.9 \%$ \\
\hline
\end{tabular}


In Fig. 4 it can be seen that the estimation error of the ADC offset is in fact null, as demonstrated earlier.

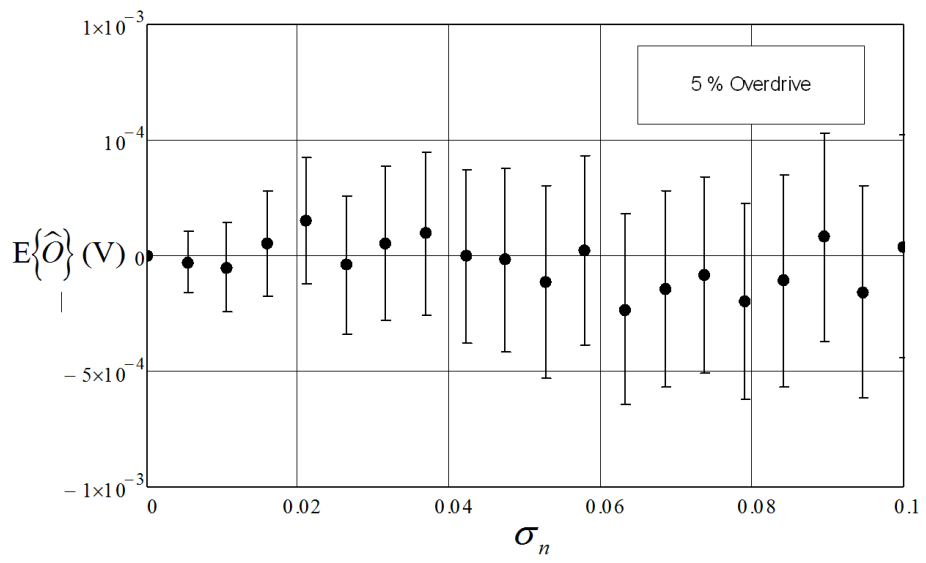

Fig. 4. Expected value of the estimated ADC offset error as a function of the normalized additive noise standard deviation for a stimulus signal amplitude of $1.05 \mathrm{~V}$ ( $5 \%$ overdrive). The circles represent the values obtained numerically. The vertical bars represent the confidence intervals for a $99.9 \%$ confidence level.

\section{Experimental Results}

In order to evaluate further the derivations presented earlier and compare them with the numerical simulation results, experimental validation was performed using the same test parameters listed in Table 1. Although many factors can affect the accuracy of ADC testing, we considered that all these factors are negligible except noise. The experimental setup consists of a data acquisition board attached to a host PC. Two signal generators were used. The first one acts as the signal source which produces the stimulus signal. The second one acts as the noise source to model additive noise in the system. The outputs of these signal generators were then connected to two separate ports on the data acquisition board using coax cables about one meter long. The data acquisition board is attached to the host PC through the PCI Bus. The host PC controls the signal generators through a USB interface. Table 2 shows the specifications of the test setup.

Table 2. Specifications of the experimental test setup.

\begin{tabular}{ll}
\hline Component & Specifications \\
\hline Host PC & CPU: Pentium 4, 3GHz, OS: Windows XP, Memory: 2GB RAM \\
\hline Signal generators & $\begin{array}{l}\text { Agilent 33210A - 10MHz Function/Arbitrary Waveform Generator } \\
\text { (calibrated) [18] }\end{array}$ \\
Data Acquisition System & $\begin{array}{l}\text { Hardware: National Instrument PCI-6220 (not calibrated) } \\
\text { Software: National Instrument Labview 8.6.1 }\end{array}$ \\
Cables & RG58 coaxial \\
Connectors & BNC type \\
\hline
\end{tabular}

In Fig. 5, it can be seen that for the case of 5\% overdrive, the experimental ADC gain measurements conform to the values calculated by the estimator such that the curve representing expression (13) follows closely the experimental curve and lies within the confidence intervals. 


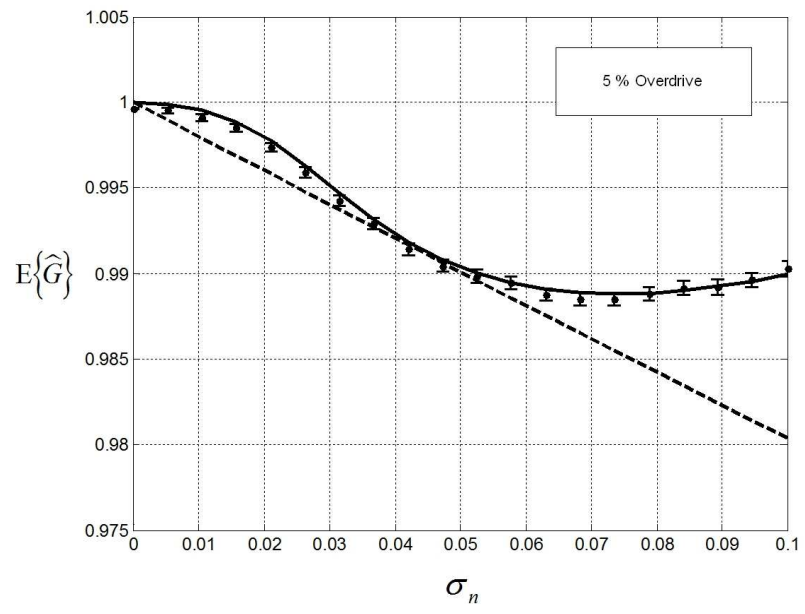

Fig. 5. Expected value of the estimated ADC gain as a function of the normalized additive noise standard deviation for a stimulus signal amplitude of $1.05 \mathrm{~V}$. The circles represent the values measured experimentally.

The vertical bars represent the confidence intervals for a $99.9 \%$ confidence level. The solid line is the representation of expression (12) and the dashed line is the representation of (17).

We observe that we get very high accuracy in the experimental results such that the theoretical curve matches the experimental results closely justifying our initial premise that only the additive noise that we introduced into the system is the primary source of uncertainty.

Fig. 6 shows that the estimation error of the terminal based offset for $5 \%$ is in fact negligible which agrees with the analytical result. Without loss of generality, we state that the estimator for the terminal based offset is unbiased by the presence of additive noise.

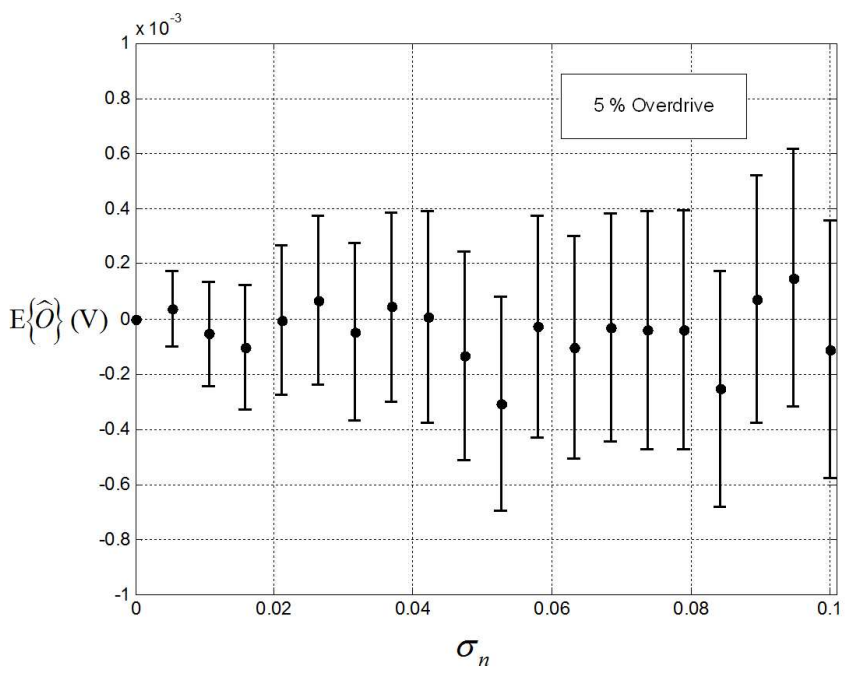

Fig. 6. Expected value of the estimated ADC offset error as a function of the normalized additive noise standard deviation for a stimulus signal amplitude of $1.05 \mathrm{~V}$ (5\% overdrive). The circles represent the values obtained numerically. The vertical bars represent the confidence intervals for a $99.9 \%$ confidence level.

\section{Conclusions}

In this paper it was shown that, using the Histogram Method, the presence of additive noise causes a bias in the estimation of the terminal based gain of an ADC but does not cause a bias in the offset error estimation.

Two analytical expressions were derived to compute the estimation error. The first, expression (13), allows the calculations of the expected value of the estimated gain with a 
high degree of accuracy when the additive noise is less than $10 \%$ of the stimulus signal. Usually the additive noise is much lower than $10 \%$ so this expression is quite accurate - the lower the noise the better. It is however not a closed form expression and thus it is difficult to use. However, it is valuable when accuracy is essential. The second one, expression (20), is a simpler expression that provides an upper bound on the relative error of the estimation. From this expression, we derived a rule-of-thumb that the relative error of the estimation is lower than one fifth of the noise standard deviation relative to the ADC full scale.

Therefore, the analytical expressions derived in this study were shown to be accurate, based on numerical simulation as well as experimental validation.

\section{Acknowledgments}

This work was sponsored by the Portuguese national research project entitled "InADC - In Depth Analysis of Analog to Digital Converter Test Methods", reference PTDC/EEA ELC/ 68843/2006 whose support the author gratefully acknowledges. Nestor Tiglao acknowledges the support of DOST and University of the Philippines through the ERDT Scholarship Grant.

\section{References}

[1] IEEE. IEEE Standard for Digitizing Waveform Recorders - IEEE Std 1057-2007 (April 2008). Institute of Electrical and Electronics Engineers. Inc.

[2] Carbone, P., Petri, D. (2000). Design of ADC sinewave histogram test. Computer Standards \& Interfaces, 22, 239-244.

[3] Blair, J. (1994). Histogram Measurement of ADC Non-linearities Using Sine Waves. In IEEE Transactions on Instrumentation and Measurement, 43(3), 373-383.

[4] Chiorboli, G., Morandi, C. (2000). About the number of records to be acquired for histogram testing of ArD converters using synchronous sinewave and clock generators. Computer Standard \& Interfaces, 22, 253-259.

[5] Corrêa Alegria, F., Cruz Serra, A. (2004). Error in the Estimation of Transition Voltages with the Standard Histogram Test of ADCs. Measurement. Elsevier Science, 35(4), 389-397.

[6] Löhning, M., Fettweis, G. (2007). The effects of aperture jitter and clock jitter in wideband ADCs. Computer Standards \& Interfaces, 29, 11-18.

[7] Arpaia, P., Daponte, P., Rapuano, S. (2003). Characterization of digitizer timebase jitter by means of the Allan variance. Computer Standards \& Interfaces, 25, 15-22.

[8] Corrêa Alegria, F., Cruz Serra, A. (April 24-27, 2006). The Histogram Test of ADCs is Unbiased by Phase Noise. IMTC 2006 - Instrumentation and Measurement Technology Conference. Sorrento. Italy, $1639-1642$

[9] Corrêa Alegria, F., Cruz Serra, A. (2001). Influence of Frequency Errors in the Variance of the Cumulative Histogram. IEEE Transactions on Instrumentation and Measurements, 50(2), 461-464.

[10] Corrêa Alegria, F., Cruz Serra, A. (2003). Variance of the Cumulative Histogram of ADCs due to Frequency Errors. IEEE Transactions on Instrumentation and Measurements, 52(1), 69-74.

[11] Attivissimo, F., Giaquinto, N., Savino, M. (2007). Worst-case uncertainty measurement in ADC-based instruments. Computer Standards \& Interfaces, 29, 5-10.

[12] Giaquinto, N., Savino, M., Trotta, A. (1998). Metrological qualification of data acquisition systems. Computer Standards \& Interfaces, 19, 219-229.

[13] Corrêa Alegria, F. (6-11 September 2009). Bias In ADC Terminal Based Gain and Offset Estimation Using the Histogram Method. Proceedings of the XIX IMEKO World Congress. Lisbon. Portugal, 710713.

[14] Corrêa Alegria F., Cruz Serra, A. (2006). ADC Transfer Function Types - A Review. Computer Standards \& Interfaces. Elsevier, 28(5), 553-559. 
[15] Corrêa Alegria, F., Cruz Serra, A. (2007). Standard Histogram Test Precision of ADC Gain and Offset Error Estimation. IEEE Transactions on Instrumentation and Measurement, 56(5), 1527-1531.

[16] Papoulis, A. (1991). Probability, Random Variables and Stochastic Processes. McGraw-Hill. $3^{\text {rd }}$ edition.

[17] Corrêa Alegria, F., Cruz Serra, A. (2004). Error in the Estimation of Transition Voltages with the Standard Histogram Test of ADCs. In Measurement. Elsevier Science, 35(4), 389-397.

[18] “Agilent 33210A 10 MHz Function/Arbitrary Waveform Generator Data Sheet”. (2008). 